\title{
Multiple Inflammatory Aortic Pseudoaneurysms
}

Meena P Rao*, Lauren Cooper, G. Chad Hughes, Lynne M. Hurwitz and Thomas M. Bashore

Duke University Medical Center, Durham, North Carolina, USA

*Corresponding author: Meena Rao, Duke University Medical Center, Erwin Road, Durham, NC 27710, USA, Tel: (o)- 919-684-24077; E-mail: meena.rao@duke.edu Received date: July 07, 2015; Accepted date: July 28, 2015; Published date: July 30, 2015

Copyright: (c) 2015 Rao MP. This is an open-access article distributed under the terms of the Creative Commons Attribution License, which permits unrestricted use, distribution, and reproduction in any medium, provided the original author and source are credited.

\section{Abstract}

Multiple mycotic pseudoaneurysms of the aorta are rarely seen. This case summarizes a patient who survived primary rupture of his proximal ascending aortic pseudoaneurysm but was an unacceptable candidate for surgical therapy. He survived for approximately four months prior to death from massive hemoptysis. The case presents a rare look at an unusual condition and the difficulties in the management of these patients.

Keywords: Mycotic pseudoaneurysm; Rupture

\section{Case Presentation}

Mycotic ascending aortic pseudoaneurysms are extremely uncommon and multiple aneurysms in a single patient have rarely been reported in the literature. Early diagnosis of this condition is important given the high mortality associated with spontaneous rupture. Definitive treatment is surgical repair of the aorta.

A 58 year old Haitian male presented to an outside facility with chest pain and fever. His past medical history included hypertension, coronary artery disease (diagnosed after a medically managed myocardial infarction), ischemic stroke, and tobacco abuse. He had suffered a motor vehicle collision 27 years prior resulting in a mandibular fracture treated with maxillomandibular fixation. The wires were never removed, and he fed himself through a gap in his teeth.

On initial presentation, his electrocardiogram revealed greater than $2 \mathrm{~mm}$ ST segment elevation in the inferior leads and leads V1-4. Emergent cardiac catheterization revealed a $90 \%$ left anterior descending artery (LAD) lesion, and a bare metal stent was successfully placed. A ventriculogram revealed normal LV function and no mitral regurgitation. Significant calcification of the ascending aorta was noted (Figure 1). Laboratory results were remarkable for a leukocytosis. Blood cultures were significant for methicillin-resistant staphylococcus aureus (MRSA) bacteremia. A subsequent computed tomography (CT) scan of chest, abdomen and pelvis did not reveal a source of infection. An echocardiogram was performed and reported as normal. The patient was treated for uncomplicated MRSA bacteremia with broad-spectrum intravenous antibiotics for 16 days and discharged home with resolution of his symptoms.

Approximately six weeks after discharge, the patient had recurrence of his chest pain and returned to the local emergency department. The electrocardiogram revealed significant $2 \mathrm{~mm}$ ST segment elevation in leads V2-4 and repeat emergent coronary angiography revealed a patent LAD stent without other coronary artery disease. Significant laboratory data included a leukocytosis with $75 \%$ polymorphonuclear neutrophils, an elevated erythrocyte sediment rate and C-reactive protein, and an elevated troponin and CK-MB. Blood cultures were negative. An echocardiogram was interpreted as abnormal with new atrial septal thickening, a large circumferential pericardial effusion, and the suggestion of a mass in both the right atrium and left atrium. The patient was then transferred to our facility for further evaluation of the cardiac mass.

On arrival, his physical exam was significant for a blood pressure of 109/57 mm Hg without a paradox, heart rate of 77 beats/min and regular and a temperature of 36.3 degrees $C$. There was no evidence of right heart pressure elevation or loss of pulses. A chest wall echocardiogram revealed an echo-lucent mass in the area of the transverse pericardial sinus with extension into anterior pericardium, left atrium and right atrium suggesting a cardiac tumor (Figures 2 and 3). A magnetic resonance imaging was not feasible due to the jaw wires. A CT scan was obtained that showed multiple aortic pseudoaneurysms involving the ascending aorta, inferior aspect of the aortic arch and the right subclavian artery. The masses seen on the chest wall echocardiogram were related to a massive pericardial hematoma seen on CT images (Figures 4 and 5).

A workup was undertaken to understand the etiology of the multiple aortic pseudoaneurysms. There was little evidence for vasculitis with a negative rheumatologic work up including rheumatoid factor, Anti ds DNA, Anti smooth muscle Ab and ANA. HIV, syphilis and tuberculosis were all ruled out. Repeat blood cultures were negative. The normal appearance of the ascending aorta at the time of cardiac catheterization suggests the staphylococcal bacteremia may have been causal, resulting in multiple mycotic aneurysmal formations with rupture into the pericardium. Remarkably, the aortic rupture did not result in acute tamponade and death.

Cardiothoracic surgery was consulted for repair of the pseudoaneurysms. Given the involvement of the ascending aorta, inferior aspect of the aortic arch and the right subclavian artery, repair would require ascending aorta and total arch replacement. It was felt his operative risk would be unacceptably high due to associated porcelain aorta, need for tracheostomy given his permanently wired jaw as well as severe malnutrition. The options were discussed with the patient and his caregivers, and he was eventually discharged to home hospice. Approximately four weeks after discharge from our facility, the patient developed massive hemoptysis and died suddenly in hospice care. 
Citation: Rao MP, Cooper L, Hughes GC, Hurwitz LM, Bashore TM (2015) Multiple Inflammatory Aortic Pseudoaneurysms. J Cardiovasc Dis Diagn 3: 213. doi:10.4172/2329-9517.1000213

Page 2 of 3

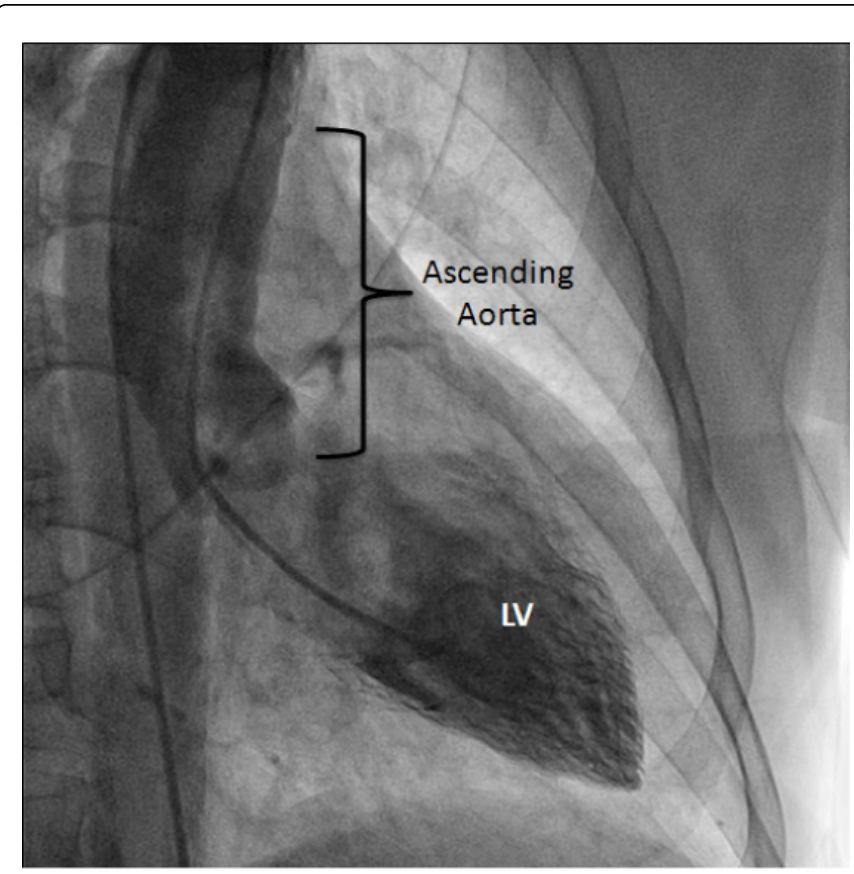

Figure 1: Ventriculogram at the time of initial presentation of chest pain with subsequent cardiac catheterization. The aorta is noted to be heavily calcified but no aneurysms or dissections are seen. $L V=$ left ventricle.

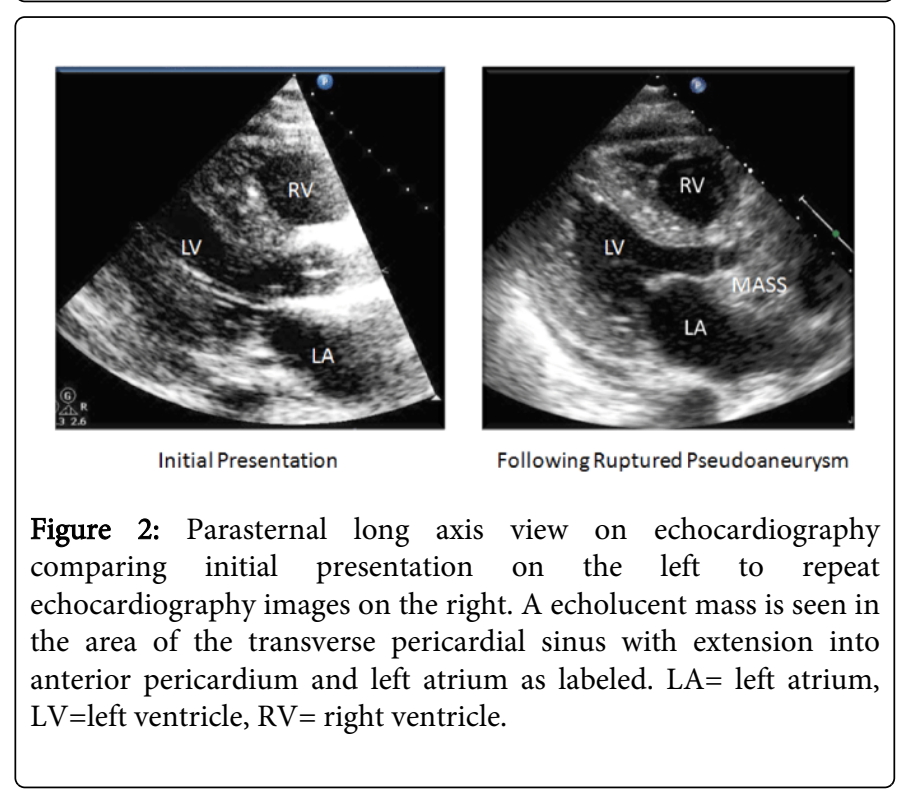

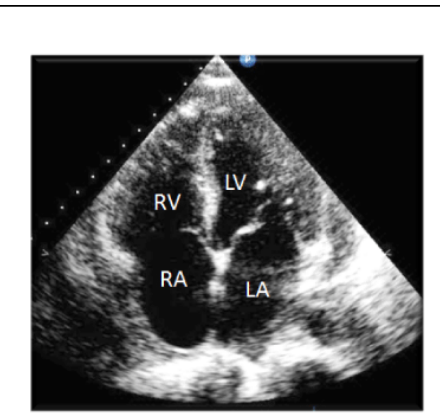

Initial Presentation

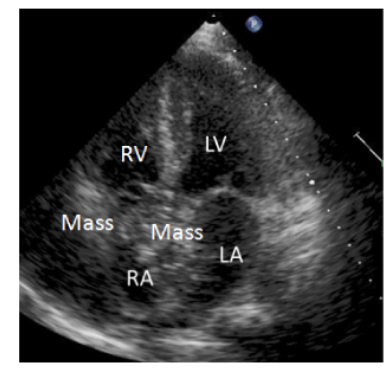

Following Ruptured Pseudoaneurysm
Figure 3: Apical four chamber view on echocardiography comparing initial presentation on the left to repeat echocardiography images on the right. Here the echo lucent mass is seen in pericardial space and both the left and right atrium as labeled. $\mathrm{LA}=$ left atrium, $\mathrm{LV}=$ left ventricle, $\mathrm{RA}=$ right atrium, $\mathrm{RV}=$ right ventricle.

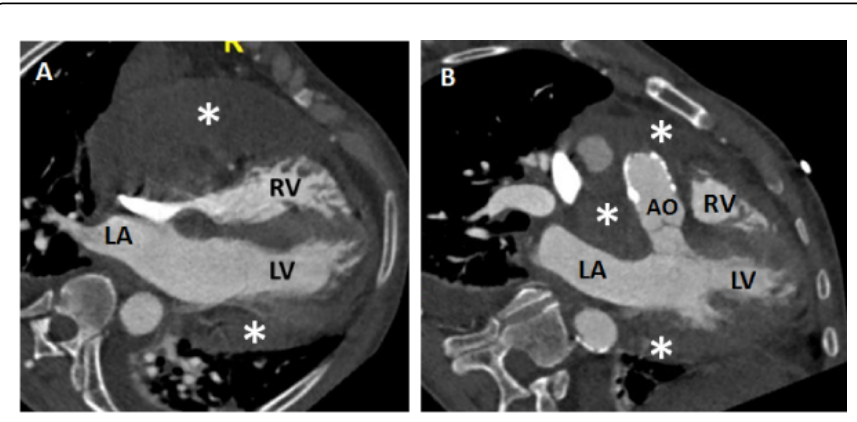

Figure 4: Coronal oblique and axial CTA images of the thorax demonstrating large pericardial hematoma $\left.{ }^{*}\right)$. Aortic calcification is also seen on side $\mathrm{B}$. Ao $=$ Aorta, $\mathrm{LA}=$ left atrium, $\mathrm{LV}=\mathrm{left}$ ventricle, $\mathrm{RV}=$ right ventricle.
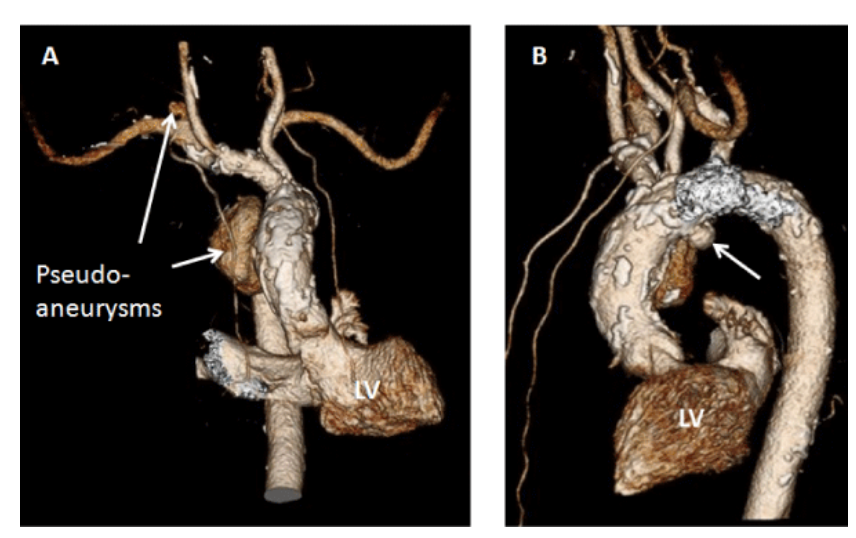

Figure 5: 3D volume rendered computed tomography reconstruction demonstrating pseudoaneurysm off ascending aorta, posterior outpouching off proximal right subclavian artery. Another pseudoaneursym is seen along the inferior aspect of the aortic arch in the image on the right. $L V=$ left ventricle. 
Citation: Rao MP, Cooper L, Hughes GC, Hurwitz LM, Bashore TM (2015) Multiple Inflammatory Aortic Pseudoaneurysms. J Cardiovasc Dis

Page 3 of 3

\section{Discussion}

Mycotic pseudoaneurysms are rare and account for only $0.7-1.3 \%$ of all aortic aneurysms [1]. Of these, most are in the abdominal aorta and only approximately $15 \%$ are reported in the ascending thoracic aorta [2]. Most mycotic pseudoaneurysms of the ascending aorta are caused by bacterial infection with common pathogens including S. aureus, S. epidermidis, salmonella and other staphylococcus [3]. Prior to aggressive antibiotic therapy, frequent causes of mycotic pseudoaneurysms of the ascending aorta included direct extension of infection from bacterial endocarditis or infection with syphilis causing destruction of the vasa vasorum and subsequent degeneration of medial elastic fibers [4]. Arteritis (Takayasu's arteritis, giant cell or granulomatous arteritis associated with polymyalgia rheumatica) can also result in pseudoaneurysm of the ascending aorta by medial destruction from inflammation [5]. Other causes of ascending aortic pseudoaneurysm include atherosclerosis, acute aortic injury, chronic traumatic injury and post-cardiac surgery patients who develop pseudoaneurysm along suture lines or cannulation sites in the aorta [6]. In 1997, only 42 cases of ascending aortic pseudoaneurysm due to bacterial infection without endocarditis had been described [7]. Of those cases, only 2 patients had more than one pseudoaneurysm involving the ascending aorta.

Mycotic pseudoaneurysms are important due to the poor prognosis associated with rupture. Ascending aortic pseudoaneurysms due to intrinsic aortic medial disease enlarge an average of 3 to $5 \mathrm{~mm} / \mathrm{yr}$ with the median diameter at aneurysm rupture being $6 \mathrm{~cm}$ [8]. Untreated pseudoaneurysms have a survival rate of $65 \%$ at 1 year and $20 \%$ at 5 years. Surgical repair of a pseudoaneurysm prior to rupture improves outcome however the mortality rate is estimated at 5 to $10 \%$ at 30 days and is 40 to $50 \%$ at 10 years [8]. Untreated ruptured pseudoaneurysms are universally fatal. Our patient is the first described in the literature to survive initial rupture and without surgical treatment he lived for four months after initial presentation.

\section{Disclosures}

None

\section{References}

1. Nagano N, Yamamoto T, Amano A, Kikuchi K (2010) Infected aneurysm of the aortic arch with purulent pericarditis caused by Streptococcus pneumoniae. Interact Cardiovasc Thorac Surg 10: 459-461.

2. Yeluri SV, Vaidya AB, Patel HJ, Kapadia SR, Karanth S (2004) Ruptured chronic traumatic mycotic pseudoaneurysm of the ascending aorta. Asian Cardiovasc Thorac Ann 12: 254-256.

3. Mukherjee JT, Nautiyal A, Labib SB (2012) Mycotic aneurysms of the ascending aorta in the absence of endocarditis. Tex Heart Inst J 39: 692-695.

4. Robbins SL, Cotran RS, Kumar V (1984) Blood vessels In Robbins SL, Cotran RS, Kumar V (eds): Pathologic Basis of Disease, 3rd ed. Philadelphia, WB Saunders: 503.

5. Petersen IM, Gunthaner DF (1986) Aortic pseudoaneurysm complicating Takayasu disease: CT appearance. J Comput Assist Tomogr 10: 676-678.

6. Sato O, Tada Y, Miyata T, Shindo S (1992) False aneurysms after aortic operations. J Cardiovasc Surg (Torino) 33: 604-608.

7. Chen YF, Lin PY, Yen HW, Lin CC (1997) Double mycotic aneurysms of the ascending aorta. Ann Thorac Surg 63: 529-531.

8. Porter RS, Kaplan JL et al. (2013) Diseases of the Aorta and its Branches: Aortic aneurysms. Merk Manual Online. 